hauptsächlich in den USA gebräuchlich sind, bei denen das $\Delta \mathrm{pH} / \mathrm{Std}$. nur 0,2 $\mathrm{pH}$-Einheiten (mit einem angeblichen Fehler von $\pm 0,02$ bis $0,03 \Delta \mathrm{pH} / \mathrm{Std}$.) angegeben wird (20). Eine der gebräuchlichsten Methoden stammt von RIDER und Mitarbeitern (21). Es ist unschwer einzusehen, daß die Eigenverseifung der verwendeten Substrate als Fehler in das Resultat eingeht.

Eine weitere, besonders in den USA sehr verbreitete Schnellmethode beruht auf der Tatsache, daß die enzymatisch aus den jeweils verwendeten Cholinestern in Freiheit gesetzten Säuren einen pH-Abfall bewirken, der mit Hilfe eines Indikators angezeigt wird (22). Meist wird die Zeit vom Zusammenbringen des biologischen Materials mit dem Substrat bis zum Umschlag des
Indikators gemessen und als Maß für die Cholinesteraseaktivität bewertet. Ein ähnliches Verfahren wurde 1959 auch in Europa empfohlen, konnte sich aber nicht recht durchsetzen (23). Bei den meisten der erwähnten Methoden geht die Eigenverseifung der Substrate als Enzymaktivität in das Resultat ein. Dagegen geht bei allen von uns bekanntgegebenen Methoden und deren Varianten (z. B. (10,14, 24)) die Eigenverseifung der Substrate nicht in das Resultat ein. Die gefundenen Ergebnisse sind unverfälschte Enzymaktivitäten.

Welche Bedeutung der Eigenhydrolyse der Cholinester bei der Übertragung von Impulsen zwischen Nervenendplatten und Muskel zukommen, muß weiteren Untersuchungen vorbehalten bleiben.

\title{
Literatur
}

1. Goedde, H. W., D. Gehring und R. Hofmann, Z. analyt. Chem. 212, 238 (1965). - 2. Kalow, W. und K. Genest, Canad. J. Biochem. Physiol. 35, 339 (1957). - 3. Harris, H. und M. WhitTAKER, Ann. hum. Genet. 26, 59 (1962). - 4. Harris, H. und M. Whittaker, Nature (London) 191, 4787 (1961). - 5. Hofstee, B. H., Science (Lancaster, Pa.) 114, 128 (1951). - 6. Pruz, W., Z. analyt. Chem., 162, 81 (1958); 166, 189 (1959). - 7. PrLz, W. und I. Johann, Z. analyt. Chem. 315, 105 (1965). - 8. Kalow, W., Anesthesiology 20, 505 (1959). - 9. Pilz, W. und H. HörLein, Hoppe-Seyler's Z. physiol. Chem. 339, 157 (1964). - 10. Przz, W., diese Z. 3, 89 (1965). - 11. Prlz, W., H. Hörlein und E. Stelzr, Hoppe Scylers' Z. physiol. Chem., im Druck. - 12. Przz, W., Biochem. Z., im Druck. - 13. Doenicke, A., Dtsch. med. Wschr., im Druck. - 14. Pilz, W., I. Johann und E. Stelzz, Klin. Wschr. 43, 1227 (1965). - 15. Eassohn, L. H. und E. Stedman, Proc. Roy. Soc. (London) Ser. B 121, 142 (1936). - 16. Alles, G. A. und R. C. Hawes, J. biol. Chemistry 133, 375 (1940). - 17. Pilz, W., Hoppe-Seyler's Z. physiol. Chem., im Druck. - 18. Mrchex, H. O., J. Laborat. Clin. Med., S. Louis 34, 1564 (1949). - 19. FRYER,
J. H., R. G. D. Steer und H. H. Williams, Arch. industr. Health 12, 406 (1955). Stubbs, J. L. und J. I. FAbes, Amer. J. Med. Techn. 26, 25 (1960). Wolfsie, J. H. und G. D. Winter, Arch. industr. Hyg. 6, 43 (1952). - 20. Gammin, J. F., B. B. Brody und F. A. Fucrle, Medical Invest, Branch Scientific Report Nr. 3, Dugway Proving Ground, Utah, July 14, (1954). - 21. Rider, J.A., J. L. Hodges, J. Swader und A. D. Wiggins, J. Laborat. Clin. Med., S. Louis 50, 376 (1957). - 22. Davies, D. R. und J. D. Nicholls, Brit. Med. J. 1, 1373 (1955). Edson, E. F., World Crops, 10, 49 (1958). Edson, E. F. und M. L. Fenwick, Brit. Med. J. 1, 1218 (1955). Fleisher, J. H., G. S. Woodson und L. Simet, Arch. industr. Health 14, 510 (1956). Limperos, G. und K. E. Ravta, Science (Washington) 117, 453 (1953). - 23. SAILER, S. und $H$. Braunstenner, Klin. Wschr. 37, 986 (1959). - 24. Pilz, W., Klin. Wschr. 36, 1017 (1958). Prlz, W., Zschr. exper. Med. 132, 310 (1959). Pruz, W., in: Handbuch der enzymatischen Analyse, Verlag Chemie, Weinheim/Bergstr. (1962). Pilz, W. und G. Kimmerle, Hoppe-Seyler's Z. physiol. Chem. 327, 280 (1962), vgl. auch $(10,12)$.
Dr. W. Pilz

Leiter des Physiol.-Chem. und Analyt. Labors der ärztlichen Abt. 509 Leverkusen-Bayerwerk

\section{Plasmabogen für klinisch-chemische Analysen ${ }^{1)}$}

\author{
Von R. Herrmann und K. Rötger \\ Aus der Abteilung für Medizinische Phjsite an der Universitäts-Hautklinik Gießen (Leiter: Prof. Dr. R. Herrmann)
}

(Eingegangen am 21. März 1966)

\begin{abstract}
Einleitend werden die Schwierigkeiten aufgezeigt, die zu überwinden sind, wenn man mit einfachen und schnellen flammenspektrophotometrischen Analysenmethoden möglichst viele Elementkonzentrationen in biologischen Lösungen analysieren will. Bei den schwerer dissoziierbaren Bestandteilen ist die Anwendung größerer Energien bzw. höherer Temperaturen nötig. Hierfür bietet sich der Plasmabogen an. Es wird eine bei Untersuchungen an biologischen Lösungen bewährte Modifikation eines geeigneten Brenners beschrieben und es werden damit bestimmte chemische Nachweisgrenzen angegeben.

If a simple and rapid flame photometric method is required for the determination of as many elements as possible in biological fluids, certain difficulties must be overcome; these are discussed. With the less dissociable components, greater energies or higher temperatures are necessary. The plasma arc can be used for this purpose. A modified burner, which can be used for studies on biological solutions, is described and its limits of chemical detection are given.
\end{abstract}

Emissionsflammenphotometrische Analysenmethoden haben sich für die Bestimmung der Elementkonzentrationen von Natrium, Kalium und Calcium in Körper-

1) Unterstützt mit Mitteln der Deutschen Forschungsgemeinschaft. flüssigkeiten dank ihrer Einfachheit, Schnelligkeit, Spezifität, geringer Streubreite und ihrer geringen Störantälligkeit in den klinisch-chemischen Laboratorien weitgehend durchgesetzt (1). Die Vorzüge dieser 
schnellen Methode lassen sich bei höherem Aufwand bei der Probenvorbereitung (z. B. vorherige Anreicherung) und/oder höherem apparativen Aufwand auf direkte Analysen einiger weiterer Elemente ausdehnen. Bestimmungsmethoden für $\mathrm{Cs}(2), \mathrm{Cu}(3,4), \mathrm{Li}(5), \mathrm{Mg}$ (6-10) und $\mathrm{Rb}$ (11) werden in den zitierten Arbeiten beschrieben.

Emissionsverfahren für die zuletzt genannten Elemente haben bis jetzt wenig Eingang in die Praxis gefunden. Darüber hinaus sind noch indirekte Bestimmungsmethoden mit Hilfe der Flamme für weitere Elemente wie z. B. Chlor angegeben worden, auf die wir hier nicht näher eingehen wollen. Noch weitere Elemente lassen sich mit diesen Flammen-Emissionsmethoden infolge der geringeren Konzentrationen in den Körperflüssigkeiten und den dafür zu schlechten Nachweisgrenzen nicht mehr analysieren. Die Gründe für diese relativ schlechten Nachweisgrenzen weiterer biologisch interessanter Elemente sind: zu hoher Energiebedarf für die Dissoziation der Verbindungen zu Atomen und zu hoher Energiebedarf für die Anregung der freien Atome (Anregung zur Emission von charakteristischen Atomlinien). Diese hohen Energien können die verhältnismäßig kühlen chemischen Flammen (etwa 1800 bis $3000^{\circ}$ ) nicht oder nicht in genügendem $\mathrm{Maße}$ aufbringen.

\section{Atomabsorption}

Fortschritte hinsichtlich der Ausdehnung dieses eleganten Analysenverfahrens auf noch weitere Elemente (wie z. B. $\mathrm{Zn}, \mathrm{Hg}, \mathrm{Cd}$ u. a.) brachte das relativ junge Atomabsorptionsverfabren (Übersichtsarbeiten dazu: $(1,12-15)$ ) Hierbei braucht die Flamme die Anregungsenergie nicht aufzubringen, jedoch muß sie nach wie vor die Dissoziationsenergie überwinden. Mit dieser Methode lassen sich also solche Elemente zusätzlich analysieren, deren Anregungsenergie hoch, deren Dissoziationsenergie aber nicht zu hoch liegt. Diese sog. Absorptions-Methoden dürften immer mehr Eingang in die klinisch-chemische Praxis finden, zumal Elemente wie $\mathrm{Cu}$ und $\mathrm{Mg}$ auch damit - z. T. sogar besser als mit Emissionsmethoden analysiert werden können.

\section{Höhere Temperaturen}

Trotz dieser erzielten Fortschritte bleiben noch Wünsche nach der Entwicklung von weiteren einfachen aber spezifischen spektrochemischen Analysenmethoden für die klinische Chemie offen. Um weiterzukommen, muß man größere Energien für die Überwindung der höherliegenden Dissoziationsenergien, also höhere Temperaturen erzeugen. Temperaturen oberhalb $3000^{\circ}$ lassen sich mit "chemischen Flammen" nur schwer und mit großen Nachteilen - wie z. B. große Giftigkeit der verwandten Gase - aufbringen. Es empfiehlt sich daher, zu "physikalischen Flammen" überzugehen. Im Prinzip hat man in den bekannten Bogen- und Funken-Anregungsquellen (16-24) genügend hohe Energien für diese Aufgabe zur Verfügung. Als Nachteile dieser Verfahren sind zu nennen: hoher Zeitbedarf für die Probenvorbereitung (Trocknen, Pulverisieren, Herstellen von Presslingen usw.) und mäßige Reproduzierbarkeiten der Analysenergebnisse.

\section{Plasmabogen}

Diese Nachteile kann man vermeiden, wenn man den von Owen, Margoshes, Scribner u. a. angegebenen Plasmabogen (25-30) für die oben geschilderte Aufgabe einsetzt. Es handelt sich dabei im Prinzip um einen Gleichstromkohlebogen, in den die flüssige Analysenprobe, ähnlich wie bei der vorgenannten Emissions- und Absorptions-Flammenphotometrie, mit Hilfe eines Zerstäubers gut reproduzierbar durch Zerstäuben eingebracht' wird. Dieser Plasmabogen ist unseres Wissens für klinisch-chemische Untersuchungen noch nicht eingesetzt worden. Hier soll die Bauweise dieses für klinische Anwendungen in unserem Laboratorium modifizierten Brenners kurz beschrieben werden (Abb. 1). Im Anschluß daran werden einige mit diesem Bogen erzielte Nachweisgrenzen angegeben.

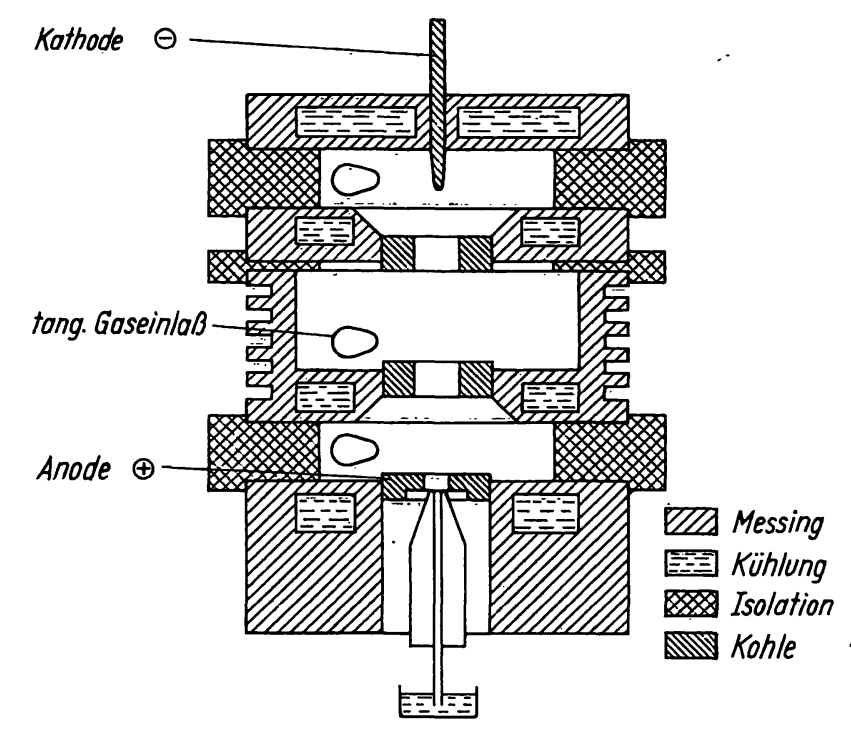

$\cdots$

Abb. 1

Brenner für Dreikammer-Plasmabogen, Modifikation nach RöTGER

Zwischen einer wassergekühlten, ringförmigen Anode aus spektralreiner Kohle und einer ebenfalls wassergekühlten Stabelektrode aus thoriertem Wolfram als Kathode wird ein Lichtbogen gezündet. Der zylindrische Raum zwischen beiden Elektroden wird durch zwei kreisförmige Stabilisierungsblenden aus reiner Kohle in drei Kammern geteilt. In jeder Kammer befindet sich eine tangentiale Gaseinblasöffnung, durch die das Betriebsgas, bei unseren Versuchen Schweißargon, zur Zusammenschnürung und somit Stabilisierung des in der Längsachse brennenden elektrischen Lichtbogens eingeblasen wird. Die hohe Geschwindigkeit des tangential strömenden Argons verhindert außerdem, da B Analysentröpfchen aus dem Innern an die Kammerwände kommen können, was sonst zu Störungen durch Verschleppungseffekte beim Wechsel der Proben führen würde. Die Analysenlösung wird, ähnlich wie bei einem Flammenphotometer, von unten in Form von Tröpfchen mittels eines Zerstäubers zugeführt. Das Treibgas für 'den Zerstäuber ist ebenfalls Schweißargon.

Bei der von uns gewählten Modifikation brennt die obere WolframStabelektrode (Kathode) nicht frei in Luft, sondern in einer von Argon durchströmten Kammer. Das bringt folgende Vorteile: Die Elektrode brennt praktisch nicht ab, es entfällt das lästige Nachschieben des Elektrodenstabes und es entfallen die durch das ungleichmäßige Nachschieben verursachten Intensitätsschwankungen. 


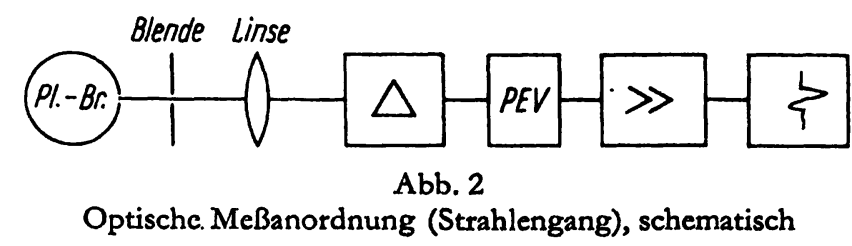

Die in Abbildung 2 gezeigte Blende ermöglicht es, einen bestimmten Teil der Plasmasäule zur Messung auszublenden. Als Spektralphotometer verwendeten wir das Modell "DU 2400" der Fa. Beckman mit einem Quarzprisma als Monochromator und einem PhotoSekundär-Elektronen-Vervielfacher PEV der Fa. RCA, Modell „1 P 28“ als Strahlungsempfänger. Über den eingebauten Gleichstromverstärker wurde das zeitliche Verhalten der Signale mit einem Honeywell-Schreiber registriert. Gemessen wird die Linienemission des in das Plasma hineingeblasenen Analysenmaterials durch eine Beobachtungsöffnung in der mittleren Kammer. Die Gesamtmeßanordnung zeigt schematisch Abbildung 2.

\section{Nachweisgrenzen}

Um einen Überblick über die Einsatzmöglichkeit dieses Plasmabrenners für klinisch-chemische Untersuchungen zu bekommen, wurden die Nachweisgrenzen bei 23 Elementen in wäßrigen Lösungen bestimmt (s. Tab. 1). Die günstigsten Betriebsbedingungen des Plasmabrenners wurden ermittelt, indem das Verhältnis Linie zu Untergrund bei verschiedenen Elementen graphisch aufgetragen wurde und zwar in Abhängigkeit von der Stromstärke, von der Gasdurchflußmenge und von der Meßhöhe. Näheres dazu soll später an anderer Stelle veröffentlicht werden. Der Gesamtgasdurchsatz betrug bei diesen Messungen etwa 43,5 l/Min., worin 3,5 l/Min. Zerstäubergas enthalten sind. Die Kühlwasserdurchflußmenge betrug 1,5//Min.

Rechnet man $1 / 2$ Min. Zeitbedarf für eine Analyse und 1,6 Pfg. Kosten je / Gas, so entstehen etwa 35 Pfg. Gaskosten für jede Analyse.
Tab. 1

Nachweisgrenze für einige Elemente in wäßr. Lösungen mit dem Drei-Kammer-Plasmabogen

\begin{tabular}{lcc}
\hline Element & $\begin{array}{c}\text { Nachweisgrenze } \\
(\text { ppm) }\end{array}$ & $\begin{array}{c}\text { Benutzte } \\
\text { Wellenlänge }(\mathrm{m} \mu)\end{array}$ \\
\hline $\mathrm{Ag}$ & 3,0 & 211,38 \\
$\mathrm{Al}$ & 6,0 & 309,27 \\
$\mathrm{As}$ & 1,0 & 195,7 \\
$\mathrm{~B}$ & 0,1 & 249,7 \\
$\mathrm{Ba}$ & 2,0 & 230,4 \\
$\mathrm{Be}$ & 0,05 & 313,0 \\
$\mathrm{Bi}$ & 100 & 289,79 \\
$\mathrm{Cd}$ & 0,4 & 214,44 \\
$\mathrm{Co}$ & 1,0 & 238,8 \\
$\mathrm{Cr}$ & 0,1 & 205,55 \\
$\mathrm{Fe}$ & 1,0 & 259,9 \\
$\mathrm{Hg}$ & 0,1 & 194,23 \\
$\mathrm{Mn}$ & 0,1 & 257,6 \\
$\mathrm{Mo}$ & 0,1 & 284,8 \\
$\mathrm{Ni}$ & 2,5 & 231,7 \\
$\mathrm{P}$ & 0,5 & 213,6 \\
$\mathrm{~Pb}$ & 0,8 & 220,3 \\
$\mathrm{Sb}$ & 3,0 & 206,8 \\
$\mathrm{Se}$ & 1,0 & 196,0 \\
$\mathrm{Si}$ & 0,1 & 251,6 \\
$\mathrm{Sn}$ & 5,0 & 189,9 \\
$\mathrm{Sr}$ & 0,05 & 215,284 \\
$\mathrm{Te}$ & 5,0 & 214,27 \\
$\mathrm{Ti}$ & 0,1 & 323,45 \\
$\mathrm{~V}$ & 1,5 & 309,3 \\
$\mathrm{Zn}$ & 0,1 & 202,5 \\
\hline & & \\
& &
\end{tabular}

Von allen in der Tabelle 1 angegebenen Elementen wurden nach der Methode von KaISER (31) Nachweisgrenzen bei verschiedenen Emissionslinien bestimmt. Es wurden in der Tabelle allerdings nur diejenigen Nachweisgrenzen angegeben, die bei unserer Meßanordnung die günstigsten Werte lieferten.

Die angegebenen Nachweisgrenzen zeigen, daß mit dem hier dargestellten Plasmabrenner neue einfache Möglichkeiten für die klinisch-chemische Anwendung der Spektralanalyse eröffnet werden.

\section{Literatur}

1. Herrmann, R., Z. analyt. Chem. 212, 1 (1965). - 2. Feldman, C. und T. C. RaIns, Analytic. Chem. 36, 405 (1964). - 3. BeaUchene, R. E., A. D. Berneking, W. G. Schrenk, H. L. MitCHell und R. E. Silker, J. biol. Chemistry 214, 731 (1955). - 4. Herratann, R., Zschr. ges. exp. Med. 126, 334 (1955). - 5. Lang, W. und R. HerritanN, Zschr. exper. Med. 139, 200 (1965). - 6. Davis, S., J. biol. Chemistry 216, 643 (1955). - 7. Dawson, J. B. und D. J. Exlis, Coll. Spectr. Intern. XII, Exeter (1965). S. 271 (London)-8. FAwCETr, J.K. und V. WYNN, J.Clin. Path., London 14, 403 (1961). - 9. HerrmanN, R., Zschr. exper. Med. 126, 371 (1955). 10. Montgomery, R. D., J. Clin. Path., London 14, 400 (1961). 11. LANG, W., Zschr. exper. Med. 139, 438 (1965). - 12. HerrManN, R., diese Z. 3, 178 (1965). - 13. Perkin-Elmer, AtomAbsorptions-Spektrophotometrie im medizinischen und biologischen Labor. Analysentechnische Berichte (Bodenseewerk PERKINElmer) 1964. - 14. Slavin, W., Occup. Hlth. Rev. 17, 9 (1965). - 15. Wrutis, J. B., Clin. Chem. (New York) 11, 251 (1965). - 16. v. Cal ker, J. und B. Wischgolt, Z. analyt. Chem. 198, 107 (1963). - 17. GurrmanN, W., Chem. analit. (Warszawa) 7, 251 (1962). - 18, Margoshes, M., Spec. techn. Publ. Amer. Soc. Test.
Mater. No. 259, 46 (1959). - 19. Mrrchell, R. L., The spectrographic analysis of soils, plants and related materials. Commonwealth Bureau of Soil Science Harpenden, England T.C. 44 (1948). - 20. Monacelli, R., H. Tanaka und I. H. Yoe, Clin. chim. Acta (Amsterdam) 1, 577 (1956). - 21. PaixaO, L. M. und I. H. YoE, Clin. chim. Acta (Amsterdam) 4, 507 (1959). - 22. Pferlstrcker, K., Mikrochim. Acta (Wien) 1956, 319 - 23. Serth, W. und K. RuthaRdt, Chemische Spektralanalyse, Springer-Verlag BerlinGöttingen-Heidelberg (1958). - 24. VALBERG, L. S., J. M. Holt und J. Szirex, Analytic. Chem. 36, 790 (1964). - 25. LerNer, R., Spectrochim. Acta (London) 20, 1619 (1964). - 26. MAR. GOSHES, M., Spectrochim. Acta (London) 13, 151 (1959). - 27. Scribner, E. und M. Margoshes, Actes du IX Coll. Spectr. Intern. Lyon 1961, Paris 1962, S. 309. - 28. NeEB, K. H. und W. Gebauer, Z. analyt. Chem. 190, 1 (1962). - 29. Owers, L. E., Appl. Spectroscopy 15, 150 (1961). - 30. RaISEN, E., R. A. Carrigan, V. Razimas, W. A. Loseke und E. L. Grove, Appl. Spectroscopy 19, 2 (1965). - 31. Karser, H., Z. analyt. Chem. 209, 1 (1965).
Professor Dr. R. Herrmann Univ. Hautklinik, Abt. f. Med. Physik 63 Gießen, Gaffkystr. 14 